\title{
Diferenças Varietais nas Características Fotossintéticas de Pennisetum purpureum Schum. ${ }^{1}$
}

\section{Manoel Messias Pereira da Silva², Hernan Maldonado Vasquez ${ }^{3}$, Ricardo Enrique Bressan-Smith ${ }^{4}$, José Fernando Coelho da Silva ${ }^{5}$, Eleonora D'Avila Erbesdobler ${ }^{6}$}

\begin{abstract}
RESUMO - Avaliaram-se os teores de pigmentos fotossintéticos, a massa foliar específica (MFE) e as curvas de eficiência fotossintética em resposta a luz solar em oito genótipos de capim-elefante (cv. mineiro, CNPGL 91-01-2, CNPGL 91-02-4, CNPGL 91-19-1, cv. taiwan A-146, CNPGL 91-27-5, CNPGL 91-27-1 e CNPGL 91-10-2) selecionados de acordo com o nível de produtividade estabelecido na época das águas. As concentrações de clorofila $a(\mathrm{CHA})$, clorofila $b(\mathrm{CHB})$ e carotenóides (CRT) mostraram relação altamente positiva, bem como as relações de clorofilas $a / b$ (RAB) e de clorofila total/carotenóides (RCC). Os teores de CHA e CHB foram mais altos no genótipo taiwan A-146. Os valores de MFE observados indicaram menor acúmulo de matéria seca por unidade de área foliar para os genótipos CNPGL 91-27-5 e CNPGL 91-27-1. O estudo das curvas de eficiência fotossintética dos genótipos possibilitou a determinação da taxa respiratória no escuro (Rd), da irradiância de compensação (Ic), do rendimento quântico (f) e da assimilação fotossintética do carbono $\left(\mathrm{A}_{\max }\right)$ na saturação luminosa. Os genótipos taiwan A-146 e mineiro apresentaram, respectivamente, cerca de 18 e $11 \%$ mais capacidade carboxilativa que os demais genótipos avaliados. Os genótipos taiwan A-146 e CNPGL 9127-5 apresentaram valores de rendimento quântico (f) próximos aos valores médios citados para plantas C4. A Rd oscilou entre 1,64 e 3,48 mmol m $\mathrm{m}^{-2} \mathrm{~s}^{-1}$ e o Ic, entre 26,39 e $54,97 \mu \mathrm{mol} \mathrm{m}^{-2} \mathrm{~s}^{-1}$ nos oito genótipos. Constatou-se que, sob condições de irradiância e temperatura não-limitantes, o genótipo taiwan A-146 apresentou maior potencial fotossintético.
\end{abstract}

Palavras-chave: eficiência carboxilativa, pigmentos fotossintéticos, potencial fotossintético, rendimento quântico, respiração

\section{Varietal Differences in the Photosynthetic Characteristics of Pennisetum purpureum, Schum.}

\begin{abstract}
Photosynthetic pigment content, specific leaf mass (SLM) and carboxilative efficiency curves were obtained in response to sunlight in eight genotypes of elephantgrass selected according to productivities during the rainy season: cv. mineiro, CNPGL 91-012, CNPGL 91-02-4, CNPGL 91-19-1, cv. taiwan A-146, CNPGL 91-27-5, CNPGL 91-27-1 and CNPGL 91-10-2. Photosynthetic pigments were highly correlated, as well as chlorophyll $a / b$ ratio (ABR) and chlorophyll/carotenoid ratio (CCR). Chlorophyll $a$ and $b$ contents were highest in taiwan A-146 genotype. SLM values indicated smaller dry matter accumulation by unit area for CNPGL 91-27-5 and CNPGL 91-27-1. Establishment of carboxilative efficiency curves of the genotypes allowed the determination of dark respiration rate (Rd), light compensation point (Ic), quantum yield (f) and maximum carbon photosynthetic assimilation $\left(\mathrm{A}_{\max }\right)$ in light saturation. The taiwan A146 and mineiro genotypes presented, respectively, about $18 \%$ and $11 \%$ more carboxilative capacity that the other genotypes. Genotypes taiwan A-146 and CNPGL 91-27-5 showed values of quantum yield (f) close to the average values reported for plants C4. Dark respiration rates varied between 1.64 and $3.38 \mu \mathrm{mol} \mathrm{m}^{-2} \mathrm{~s}^{-1}$ and Ic varied from 26.39 to $54.97 \mu \mathrm{mol} \mathrm{m}^{-2} \mathrm{~s}^{-1}$ in the eight genotypes. This study revealed that, under non limiting irradiance and temperature conditions, genotype taiwan A-146 presented highest photosynthetic rates.
\end{abstract}

Key Words: carboxilative efficiency, photosynthetic pigments, photosynthetic potential, quantum yield, respiration

\section{Introdução}

O princípio básico da produção de biomassa em forrageiras é a transformação da energia solar em compostos orgânicos, via fotossíntese, para ser convertida em massa verde, inicialmente nas folhas (GOMIDE, 1973). Notadamente, essa parte da planta é utilizada como fonte de alimento no pastejo, portanto, a fisiologia da folha passa por constantes variações.
Geralmente, o conteúdo de carbono da biomassa está próximo a $40 \%$, variando muito pouco entre as espécies vegetais ou com o ambiente (AUSTIN, 1989).

PAZ e MATOS (1985) descreveram que, nem toda a matéria seca produzida pela planta é colhida, mas distribuída entre raízes, parte aérea e órgãos de armazenamento. É provável que possa haver correlação positiva entre produção e fotossíntese para a cultura em que a maior parte da planta é colhida, do

\footnotetext{
${ }^{1}$ Parte do trabalho de tese de mestrado do primeiro autor, financiado com recursos da Fundação Estadual do Norte Fluminense (FENORTE).

2 Zootecnista, M.Sc., Laboratório de Zootecnia e Nutrição Animal (LZNA), Centro de Ciências e Tecnologias Agropecuárias (CCTA), Universidade Estadual do Norte Fluminense (UENF), Av. Alberto Lamêgo, 2000, Campos dos Goytacazes, RJ. CEP:28015-620. E.mail: manel@uenf.br 3 Professor Associado do LZNA/CCTA/UENF. E.mail: hernan@uenf.br

${ }^{4}$ Professor Associado do Laboratório de Melhoramento Genético Vegetal(LMGV), Setor de Fisiologia Vegetal, CCTA/UENF. E.mail: brsmith@uenf.br

5 Professor Titular do LZNA/CCTA/UENF. E.mail: jcoelho@uenf.br

${ }^{6}$ Zootecnista, M.Sc., Laboratório de Ciências Ambientais (LCA), Centro de Biociências e Biotecnologias (CBB), UENF. E.mail: lola@uenf.br
} 
que para as culturas em que se colhe somente frutos, grãos ou outros órgãos de armazenamento. Pode-se afirmar, portanto, que os genótipos que apresentarem alta capacidade de fixar $\mathrm{CO}_{2}$ são potencialmente capazes de aumentar a eficiência do uso do carbono, resultando em aumento do seu rendimento biológico e econômico (ZELITCH, 1982).

A seleção de materiais genéticos superiores para as características fotossintéticas baseia-se na variabilidade genética da espécie (CRISWELL e SHIBLES, 1971). Há evidências da variabilidade genética em gramíneas como o trigo (AUSTIN et al., 1982) e milho (HEICHEL e MUSGRAVE, 1969), com potencial para utilização em programas de melhoramento.

O capim-elefante (Pennisetum purpureum Schum.) é uma gramínea perene de colmos robustos e crescimento ereto, com propagação vegetativa, apresentando alto potencial de produção de matéria seca, boa qualidade nutritiva e, devido a sua origem tropical, adaptou-se muito bem às condições de clima e solo de quase todo o Brasil (PASSOS, 1994). As informações varietais sobre a fotossíntese e características relacionadas são escassas para o capimelefante, o que torna imprescindível a sua análise, tendo como referência os vários materiais genéticos advindos dos bancos de germoplasma.

No presente trabalho foram avaliadas características fotossintéticas de diferentes genótipos de capim-elefante: área foliar, massa foliar específica, pigmentos fotossintéticos e atributos da curva de eficiência fotossintética, com o objetivo de discriminar os materiais genéticos mais eficientes quanto a essas características.

\section{Material e Métodos}

Mudas enraizadas de genótipos de capim-elefante foram plantadas em potes plásticos de cinco litros, sob condição de casa-de-vegetação, num solo franco-argilo-arenoso, de pH 6,3 e 55\% de saturação de bases, adubado com 50 ppm de $\mathrm{P}_{2} \mathrm{O}_{5}$ (superfosfato simples), $100 \mathrm{ppm}$ de $\mathrm{K}_{2} \mathrm{O}$ (cloreto de potássio) e 100 ppm de N (sulfato de amônio). Os vasos foram submetidos a duas irrigações diárias até o final do experimento, para manutenção da capacidade de campo do solo e pleno desenvolvimento vegetal. Os genótipos utilizados foram provenientes do Banco Ativo de Germoplasma do capim-elefante (BAGCE), localizado na Embrapa Gado de Leite. Os genótipos foram selecionados com base na produção de matéria seca do ensaio de campo realizado no município de Campos dos Goytacazes, RJ (DAHER et al., 1997). Além das duas cultivares testemunhas, mineiro e taiwan A-146, os genótipos foram selecionados de acordo com os valores de produção total de matéria seca: alta, 22773 e $17537 \mathrm{~kg} / \mathrm{ha}$, respectivamente para CNPGL 91-27-5 e CNPGL 91-27-1; média, 13804 e $13902 \mathrm{~kg} / \mathrm{ha}$, respectivamente para CNPGL 91-02-4 e CNPGL 91-01-2; baixa, 11112 e 10252 kg/ ha, respectivamente, para CNPGL 91-19-1 e CNPGL 91-10-2.

Curvas de resposta da assimilação fotossintética do carbono (A) ao fluxo de fótons fotossintéticos (FFF) foram obtidas para os genótipos, utilizando-se a luz solar como fonte luminosa. Para a determinação das trocas gasosas foliares foi utilizado o analisador de gases no infravermelho (IRGA) modelo LI-6200 (LI-COR), sob concentração ambiente de $\mathrm{CO}_{2}$ (@) $360 \mu \mathrm{mol} \mathrm{ml}^{-1}$ ) em câmara de medição de 1 litro. Foram utilizadas plantas em estádio vegetativo de crescimento, fazendo-se as medições na primeira folha totalmente expandida e de aspecto saudável, a partir do ápice do perfilho. A escolha da parte da folha para as medições foi feita a partir de testes preliminares de resposta à fotossíntese em três regiões da lâmina foliar (ápice, meio e base), confinandoas dentro da câmara foliar do IRGA e, ao final, optouse pela parte mediana da lâmina foliar para as medições definitivas.

As medidas das variações foram tomadas no período de 9 às $11 \mathrm{~h}$, durante oito dias do mês de junho de $1999(17 / 06,18 / 06,19 / 06,20 / 06,23 / 06,24 / 06,26 / 06$ e 27/06), nos quais os valores de FFF observados foram superiores a $1800 \mu \mathrm{mol} \mathrm{m} \mathrm{m}^{-2} \mathrm{~s}^{-1}$ e decrescidos por sombreamento artificial, até valores de FFF da ordem de $0 \mu \mathrm{mol} \mathrm{m}{ }^{-2} \mathrm{~s}^{-1}$.

A extração de pigmentos fotossintéticos (clorofilas e carotenóides) foi realizada seguindo a metodologia proposta por HENDRY e PRICE (1993), com modificações. No processo inicial de extração, em que a parte mediana da quarta lâmina foliar (contada a partir do ápice) de cada genótipo foi utilizada, retirouse apenas o lado esquerdo (a partir da nervura central) da lâmina foliar para essa determinação, reservando-se o lado direito da lâmina foliar para a determinação da massa foliar específica. O tecido foliar para a extração de pigmentos foi macerado em almofariz de porcelana com adição de $\mathrm{N}_{2}$ líquido. Uma pequena porção do tecido macerado foi colocada

Rev. bras. zootec., 30(6S):1975-1983, 2001 
em um tubo "Eppendorf" com capacidade para $2000 \mathrm{~mL}$ (previamente pesado em balança analítica), pesado e homogeneizado com $1000 \mathrm{~mL}$ de acetona amoniacal (acetona $81,8 \%$; H2O 18\%; NH4OH 0,2\% v/v) sob forte agitação em vortex por 10 segundos. Em seguida, o material foi centrifugado (Microcentrifuge 5415C, Eppendorf, Germany) a $10.000 \mathrm{rpm}$ por 2 minutos e retirado $500 \mathrm{~mL}$ de sobrenadante para posterior leitura em 480, 646 e $663 \mathrm{~nm}$ em espectrofotômetro SPEKOL/ZEISS UV-VIS (Zeiss, Germany). Antes da leitura, os extratos mais concentrados foram diluídos em acetona para melhor se adequarem às leituras.

A determinação da massa foliar específica (MPE) foi obtida pela retirada de cinco discos foliares de área conhecida. Os discos foram secos a $65^{\circ} \mathrm{C}$ por 48 horas e, em seguida, pesados para obtenção do peso da matéria seca dos discos pelas suas respectivas área foliares.

Foi utilizado um delineamento experimental em blocos ao acaso, com três repetições, sendo a unidade experimental composta por uma planta. Os tratamentos foram constituídos por oito genótipos de capimelefante, seis clones (híbridos intra-específicos) e duas cultivares testemunhas (Tabela 1).

A determinação da área foliar (AF) foi feita no Setor de Fisiologia Vegetal do Laboratório de Melhoramento Genético Vegetal (LMGV) do CCTA. As lâminas foliares foram destacadas dos colmos, para determinação da área foliar total de cada genótipo, utilizando-se o medidor de área foliar, modelo LI 3100 (Li-cor, USA).

Os dados obtidos para os teores de clorofila a
(CHA), clorofila b (CHB), clorofila total (CHAB), carotenóides (CRT), relação entre teores de clorofila a e b (RAB), relação entre os teores de clorofila total $(\mathrm{a}+\mathrm{b})$ e carotenóides (RCC) e massa foliar específica (MFE) foram submetidos a análise de variância. Na comparação das médias utilizou-se o teste de Tukey, a $5 \%$ de probabilidade.

Os dados foram analisados utilizando-se o programa SAEG (Sistema para análises Estatísticas e Genéticas), desenvolvido pela Universidade Federal de Viçosa, MG (EUCLYDES, 1983), pelo seguinte modelo estatístico:

$$
\mathrm{Y}_{\mathrm{ikj}}=\mu+\mathrm{t}_{\mathrm{i}}+\mathrm{b}_{\mathrm{j}}+\mathrm{e}_{\mathrm{ij}}
$$

em que: $Y_{i k}=$ observação relativa à parcela que recebeu o tratamento $i$, no bloco $j ; \mu=$ média geral; $\mathrm{ti}=$ efeito do tratamento, $\mathrm{i}=(1, \ldots, 8) ; \mathrm{b}_{\mathrm{j}}=$ efeito do bloco, $j=(1,2,3) ; e_{i j}=$ efeito do erro aleatório, normal e independente, distribuído com média 0 e variância $\sigma^{2}$, no tratamento i e no bloco j;

\section{Resultados e Discussão}

As concentrações dos pigmentos fotossintéticos, determinadas na quarta folha totalmente expandida a partir do ápice, nos oito genótipos de capim-elefante, estão apresentadas nas Figuras 1 e 2. Os maiores conteúdos das clorofilas a (CHA) e b (CHB) nas folhas dos genótipos de capim-elefante, foram encontrados no genótipo taiwan A-146, embora não tenha se apresentado significativamente maior $(\mathrm{P}<0,05)$ que a cv. mineiro e CNPGL 91-02-4, os demais apresentaram níveis intermediários desses pigmentos. Os resultados indicaram que o teor de

Tabela 1 - Identificação dos genótipos (clones híbridos intra-específicos e cultivares testemunhas) de capim-elefante, a sua origem e a classificação da produção de matéria seca (PMS)

Table 1 - Identification of genotypes (intra-specific hybrid clones and standard cultivars) of elephantgrass, origin and dry matter yield (DMY)

\begin{tabular}{|c|c|c|c|c|}
\hline $\begin{array}{l}\text { PMS* } \\
D M Y^{*}\end{array}$ & $\begin{array}{l}\text { Genótipo } \\
\text { Genotype }\end{array}$ & $\begin{array}{c}\text { Progenitor masculino } \\
\text { Male parent }\end{array}$ & & $\begin{array}{l}\text { Progenitor feminino } \\
\text { Female parent }\end{array}$ \\
\hline $\mathrm{T}$ & cv. mineiro & & & \\
\hline M & CNPGL 91-01-2 & Elefante da Colômbia & $\mathrm{x}$ & Merker \\
\hline M & CNPGL91-02-4 & Merker & $\mathrm{x}$ & Napier Volta Redonda \\
\hline B & CNPGL91-19-1 & Merker Pinda & $\mathrm{x}$ & Elefante da Colômbia \\
\hline $\mathrm{T}$ & cv. taiwan A-146 & & & \\
\hline A & CNPGL91-27-5 & Mineiro & $\mathrm{x}$ & Merker Sata Rita \\
\hline A & CNPGL91-27-1 & Mineiro & $\mathrm{x}$ & Elefante da colômbia \\
\hline B & CNPGL91-10-2 & Taiwan A-148 & $\mathrm{x}$ & Merker \\
\hline
\end{tabular}

* Produção total de matéria seca, em três cortes na época das águas (DAHER et al., 1997).

$\mathrm{A}=\mathrm{PMS}$ alta; $\mathrm{M}=\mathrm{PMS}$ média; $\mathrm{B}=\mathrm{PMS}$ baixa e T PMS testemunha.

* Yield of total dry matter after three cuttings during the rainy season (DAHER et al., 1997).

A: high DMP; M: average DMP; B: low DMP.

Rev. bras. zootec., 30(6S):1975-1983, 2001 


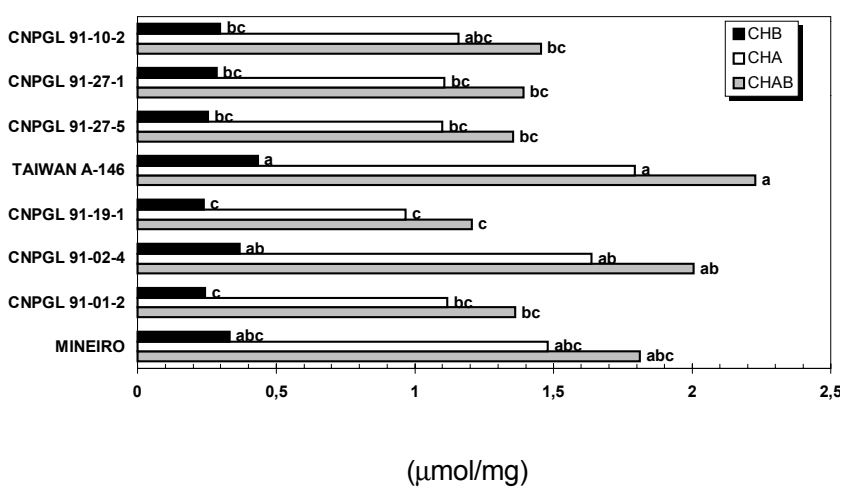

Figura 1 - Concentrações de clorofila a (CHA), clorofila $b$ $(\mathrm{CHB})$ e clorofila total (CHAB) de oito genótipos de capim-elefante. Médias seguidas da mesma letra não diferem, segundo o teste Tukey $(P \leq 0,05)$.

Figure 1 - Chlorophill a (CHA), chlorophill $b(\mathrm{CHB})$ and total chlorophill (CHAB) content of eight genotypes of elephantgrass. Means followed by the same letter are not significantly different, according to Tukey test ( $P \leq .05)$.

CHB foi, em geral, aproximadamente $1 / 4$ do teor de CHA encontrado nos genótipos, sendo inferior a relação de 1/3 descrita por HALL e RAO (1994). Esta variação é bem demonstrada quando observase a relação entre a clorofila a e a clorofila b (RAB), em que todos os genótipos apresentaram $\mathrm{RAB}$ superior a 3 (Figura 3). MONTEITH (1978), relatando algumas características fotossintéticas de plantas do grupo $\mathrm{C} 4$, encontrou um valor médio de $3,9 \pm 0,6$ para a relação clorofila a/b. Uma das características fotossintéticas das plantas de sol é demonstrar menor quantidade de moléculas de clorofila por cloroplasto, principalmente a clorofila $b$, uma vez que essas plantas não necessitam investir mais energia na produção de pigmentos coletores de energia, pois se desenvolvem otimamente em ambiente saturado de luz (SALISBURY e ROSS, 1991). Os valores máximos da concentração de clorofila total expressos por unidade de área foliar encontrados na literatura são divergentes (USUDA et al., 1984; WENG, 1984). É provável que os autores citados tenham avaliado diferentes materiais genéticos, que, conforme a Figura 1, podem apresentar substanciais diferenças.

Os genótipos de capim-elefante não apresentaram diferenças $(\mathrm{P}>0,05)$ entre seus conteúdos de carotenóides, embora tenha sido observado que incrementos na concentração de carotenóides, em geral, estão associados a aumentos na concentração de clorofila total. Tal afirmativa pode ser evidenciada

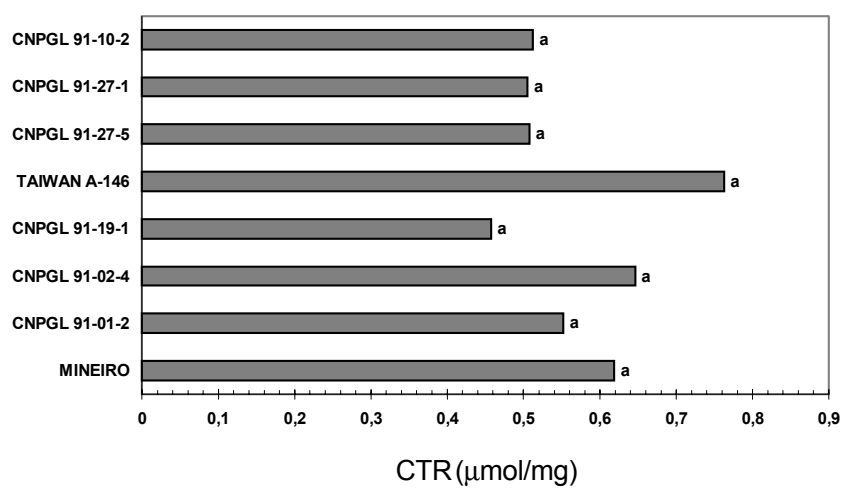

Figura 2 - Concentração de carotenóides (CRT) de oito genótipos de capim-elefante. Médias seguidas da mesma letra não diferem, segundo o teste Tukey $(P \leq 0,05)$.

Figure 2 - Carotenoid content (CRT) of eight genotypes of elephantgrass. Means followed by the same letter are not significantly different, according to Tukey test $(P \leq .05)$.

pelo teste de correlação de Pearson (Tabela 2). A alta relação entre os pigmentos reflete muito bem as funções dos carotenóides que, além de coletores de luz, são fotoprotetores, preservando as clorofilas da destruição oxidativa do $\mathrm{O}_{2}$, quando há excesso de energia captada pelas antenas coletoras de luz, sem que essa energia seja repassada aos transportadores de elétrons dos fotossistemas I e II. Segundo HENDRY e PRICE (1993), a razão entre as concentrações de carotenóides apresenta função de antioxidantes naturais, além de extinguir excitações fotoinduzidas, sendo menos sensíveis, que as clorofilas, aos ataques oxidativos e ao dano fotoquímico.

$\mathrm{Na}$ Figura 4 estão apresentados os valores da massa foliar específica (MFE), dos oito genótipos estudados. Os genótipos CNPGL 91-27-5 e CNPGL 91-27-1 diferenciaram-se significativamente dos demais, apresentando os menores $(\mathrm{P}<0,05)$ valores de MFE, enquanto os genótipos CNPGL 91-01-2 e CNPGL 91-19-1 tiveram as maiores MFE, embora o último não tenha diferido da cv. mineiro. FISCHER et al. (1998) não encontraram diferenças nos valores de MFE para oito cultivares de trigo cultivados na região noroeste do México, com valores entre 45,1 e $53,9 \mathrm{~g} . \mathrm{m}^{-2}$. A massa foliar específica varia de acordo com as diferenças na estrutura interna da folha, de tal modo que uma MFE alta, freqüentemente, resulta na acumulação de produtos secundários e do amido, rendendo, assim, maior quantidade de matéria seca

Rev. bras. zootec., 30(6S):1975-1983, 2001 
Tabela 2 - Coeficientes de correlação $(r)$ entre as características fotossintéticas: clorofila a (CHA), clorofila $b(\mathrm{CHB})$, clorofila total (CHAB), carotenóides (CRT), massa foliar específica (MFE), assimilação fotossintética máxima $\left(A_{\text {max }}\right)$, respiração escura (Rd), irradiância de compensação (Ic) e rendimento quântico $(\phi)$

Table 2 - Correlation coefficient $(r)$ among photosynthetic characteristics: chlorophyll a (CHA), chlorophyll b (CHB), chlorophyll a+b $(C H A B)$, carotenoid $(C R T)$, specific leaf weight $(S L W)$, max. photosynthetic assimilation $\left(A_{\text {max }}\right)$, dark respiration $(R d)$, light compensation point (IC) and quantum yield $(\phi)$

\begin{tabular}{lccccccccc}
\hline & CHA & CHB & CHAB & CRT & MFE & A $_{\max }$ & Rd & Ic \\
\hline CHA & 1,00 & $1,00^{*}$ & $1,00^{*}$ & $0,84^{*}$ & $-0,17$ & 0,47 & 0,60 & $-0,05$ & 0,59 \\
CHB & - & 1,00 & $1,00^{*}$ & $0,81^{*}$ & $-0,18$ & 0,45 & 0,57 & $-0,04$ & 0,57 \\
CHAB & - & - & 1,00 & $0,83^{*}$ & $-0,17$ & 0,47 & 0,59 & $-0,04$ & 0,59 \\
CRT & - & - & - & 1,00 & $-0,36$ & 0,60 & 0,64 & $-0,01$ & 0,49 \\
MFE & - & - & - & - & 1,00 & $-0,05$ & 0,09 & 0,25 & $-0,35$ \\
A $_{\max }$ & - & - & - & - & - & 1,00 & $-0,03$ & $-0,47$ & 0,55 \\
Rd & - & - & - & - & - & - & 1,00 & 0,64 & $-0,07$ \\
Ic & - & - & - & - & - & - & - & $-1,00$ & $-0,80 *$ \\
$\phi$ & - & - & - & - & - & - & - & - & 1,00 \\
\hline
\end{tabular}

${ }^{*}$ As correlações são estatisticamente significativas a $5 \%$ de probabilidade.

${ }^{*}$ The correlation coefficients are significative at $5 \%$ of probability.

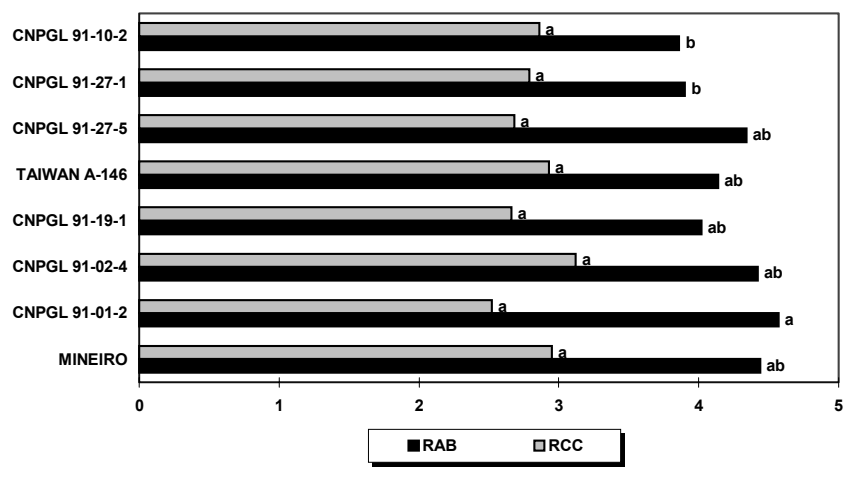

Figura 3 - Relação entre a concentração de clorofila a e a concentração de clorofila $b$ (RAB) e relação entre a concentração de clorofila total e a concentração de carotenóides (RCC), de oito genótipos de capim-elefante. Médias seguidas da mesma letra não diferem segundo o teste Tukey $(\mathrm{P} \leq 0,05)$.

Figure 3 - Chlorophyll a/b ratio and chlorophill/carotenoid ratio of eight genotypes of elephantgrass. Means followed by the same letter are not significantly different according to Tukey test $(P \leq .05)$.

por unidade de área (KONNINGS, 1990). BHAGSHARI e BROWN (1986) sugerem que variações na taxa fotossintética são devidas a diferenças na MFE. No entanto, não houve correlação entre Amax e MFE (Tabela 2), tal que o padrão dos resultados observados não foi consistente.

$\mathrm{Na}$ Figura 5 é ilustrada a curva de resposta da assimilação fotossintética do carbono (A), em função do fluxo de fótons fotossintéticos (FFF), da cv. mineiro, da

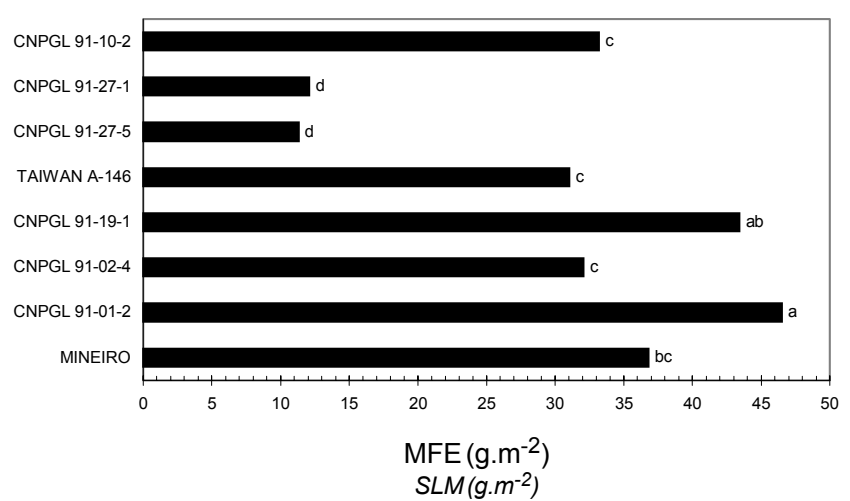

Figura 4 - Massa foliar específica (MFE) de oito genótipos de capim-elefante. Médias seguidas da mesma letra não diferem, segundo o teste Tukey $(P \leq 0,05)$.

Figure 4 - Specific leaf mass (SLM) of eight genotypes of elephantgrass. Means followed by the same letter are not significantly different according to Tukey test $(P \leq .05)$.

qual se determinaram os seguintes atributos: respiração no escuro, irradiância de compensação, eficiência quântica em baixa intensidade de luz (rendimento quântico) e ainda, a taxa de fotossíntese na saturação de luz. Esses atributos também foram determinados a partir das curvas dos demais genótipos, e os dados estão sumariados na Tabela 3 .

Na maioria das culturas, a fotossíntese aumenta linearmente em resposta ao aumento da luz incidente

Rev. bras. zootec., 30(6S):1975-1983, 2001 
até intensidades de cerca de $25 \%$ da luz solar plena. Acima desta intensidade, a taxa de fotossíntese de uma dada folha é considerada limitada pela disponibilidade de força redutora e ATP, que são fornecidos pelos processos ocorrendo nos fotossistemas I e II, necessários para recuperar o substrato da carboxilação: a enzima ribulose 1,5-bisfosfato carboxilase-oxigenase (RUBISCO). Em intensidades saturantes de luz, o processo fotoquímico não é limitado e a principal limitação da fixação de carbono é a taxa de suprimento do dióxido de carbono nos sítios de carboxilação (AUSTIN, 1989).

Observou-se que os genótipos taiwan A-146 e mineiro apresentaram, respectivamente, cerca de 18 e $11 \%$ mais capacidade carboxilativa que a média dos demais (Tabela 3). Os valores observados foram discrepantes daqueles encontrados por LUDLOW (1985) e USUDA et al. (1984), de 76 e $10 \mu \mathrm{mol} \mathrm{m}^{-2} \mathrm{~s}^{-1}$, respectivamente. As divergências entre esses valores podem ser explicadas em função das cultivares avaliadas, do estádio de desenvolvimento das mesmas e das condições em que as determinações de troca gasosa foram realizadas. O capim-elefante é uma planta $\mathrm{C}_{4}$, caracterizada pela enzima descarboxilativa NADP-málica (GUTIERREZ et al., 1976), portanto, a assimilação do $\mathrm{CO}_{2}$ não está sujeita a reduções drásticas causadas por elevações de temperatura ou por déficit hídrico moderado, como ocorre nas plantas $\mathrm{C}_{3}$.

COOPER (1970) descreveu que o capim-elefante talvez fosse a mais eficiente planta conhecida na conversão de luz solar em matéria seca, observando produção anual de matéria seca de 85,2 t/ha, em El Salvador.

Alguns dos valores de rendimento quântico $(\phi)$ da fotossíntese nessa pesquisa, como observado nos genótipos taiwan A-146 e CNPGL 91-27-5, ficaram próximos aos valores médios de rendimento quântico de $0,053 \mu \mathrm{mol} \mathrm{CO}_{2}$ fóton $^{-1}$ para espécies $\mathrm{C} 4$, relatado por EHLERINGER e BJÖRKMAN (1977). Considerando que o rendimento quântico é a medida da quantidade de $\mathrm{CO}_{2}$ fixado pela energia de fótons absorvidos pelo aparelho fotossintético, um alto rendimento quântico proporciona maior eficiência na síntese de poder assimilatório (ATP e NADPH), para posterior uso em vias anabólicas, tal como a conversão em produtos estáveis (BARRO et al., 1996).

A correlação entre a $A \max$ e o rendimento quântico dos genótipos, não foi significativa $(\mathrm{P}>0,05)$, como observado na Tabela 2. ORT e BAKER (1988) constataram que a eficiência quântica da fotossíntese, em baixa luminosidade, é sensível a alguns tipos de estresse ambiental, ao contrário do que ocorre na Amax. Segundo esses autores, a eficiência quântica é limitada em função de baixos níveis de luz incidente em folhas não expostas ao sol. O aparato fotossintético da planta é muito complexo e sujeito a fortes influências regulatórias, internas e ambientais (AUSTIN, 1989). Considera-se que, apesar de existirem marcantes diferenças entre espécies na Amax, existe pouca variação genética na eficiência quântica de folhas em baixa luminosidade. Segundo BJÖRKMAN e DEMMING (1987), o rendimento quântico, pode ser um indicador do efeito de vários estresses atuantes no sistema fotossintético.

O ponto de compensação da luz, ou Ic, revela em qual fluxo de fótons ocorre o equilíbrio entre os níveis de $\mathrm{CO}_{2}$ respirado e do $\mathrm{CO}_{2}$ absorvido pelas folhas (TAIZ e ZEIGER, 1991). A irradiância de compen-

Tabela 3 - Respiração no escuro (Rd), assimilação fotossintética máxima $\left(A_{\max }\right)$, irradiância de compensação (Ic) e rendimento quântico $(\phi)$ de oito genótipos de capim-elefante, cultivados em vasos, utilizando-se a luz solar como fonte luminosa

Table 3 - Respiration dark (Rd), maximum photosynthetic assimilation $\left(A_{\max }\right)$, light compensation point (Ic) and quantum yield ( $\left.\phi\right)$ of eight genotypes of elephantgrass, using sunlight as light source

\begin{tabular}{|c|c|c|c|c|}
\hline \multirow{2}{*}{$\begin{array}{l}\text { Genótipos } \\
\text { Genotypes }\end{array}$} & $\mathrm{Rd}$ & $\mathrm{A}_{\max }$ & \multirow{2}{*}{$\begin{array}{c}\text { Ic } \\
\left(\mathrm{mmol} \text { fóton } \mathrm{m}^{-2} \mathrm{~s}^{-1}\right) \\
\left(\mathrm{mmol} \text { photon } \mathrm{m}^{-2} \mathrm{~s}^{-1}\right)\end{array}$} & \multirow{2}{*}{$\begin{array}{c}\phi \\
\left(\mathrm{mmol} \mathrm{CO}_{2} \text { fóton }^{-1}\right) \\
\left(\mathrm{mmol} \mathrm{CO} \text { photon }^{-1}\right)\end{array}$} \\
\hline & \multicolumn{2}{|c|}{$\left(\mathrm{mmol} \mathrm{CO} \mathrm{Cm}^{-2} \mathrm{~s}^{-1}\right)$} & & \\
\hline cv. mineiro & 1,64 & 47,37 & 29,12 & 0,0381 \\
\hline CNPGL 91-01-2 & 2,02 & 39,05 & 35,10 & 0,0349 \\
\hline CNPGL 91-02-4 & 3,48 & 41,16 & 54,97 & 0,0266 \\
\hline CNPGL91-19-1 & 1,99 & 39,64 & 42,60 & 0,0261 \\
\hline cv. taiwan A-146 & 3,02 & 44,37 & 36,27 & 0,0487 \\
\hline CNPGL91-27-5 & 1,76 & 41,01 & 26,39 & 0,0457 \\
\hline CNPGL 91-27-1 & 2,19 & 40,81 & 38,36 & 0,0349 \\
\hline CNPGL 91-10-2 & 2,20 & 52,02 & 20,30 & 0,0389 \\
\hline
\end{tabular}

Rev. bras. zootec., 30(6S):1975-1983, 2001 
sação pode variar de acordo com a espécie, com a temperatura no momento da medição da concentração de $\mathrm{CO}_{2}$, mas geralmente corresponde a cerca de $2 \%$ da luz solar plena (40 $\mu$ mol fótons ${ }^{-1}$ ) (SALISBURY e ROSS, 1991). Diferenças nos pontos de compensação também são causadas por mudanças na taxa de respiração, tal que, quanto menor a taxa respiratória, menos fótons serão necessários para atingir a Ic. Entretanto, não houve correlação ( $\mathrm{P}>0,05)$ entre Ic e Rd nesse trabalho (Tabela 2), indicando que nesses genótipos a respiração não é o maior componente afetando este atributo. Ainda assim, a irradiância de compensação foi negativamente correlacionada com o rendimento quântico (Tabela 2), indicando que maiores rendimentos quânticos com menores pontos de compensação podem revelar genótipos com melhor adaptação a ambientes com baixa luminosidade.

A respiração no escuro (Rd) reflete uma condição em que, não havendo absorção da radiação luminosa pelas folhas, a quantidade de $\mathrm{CO}_{2}$ liberado pela respiração excede a de $\mathrm{CO}_{2}$ fixado na fotossíntese (LARCHER, 1995). Diferenças na taxa de respiração no escuro têm sido usadas como critério para a discriminação de linhagens com baixas taxas de

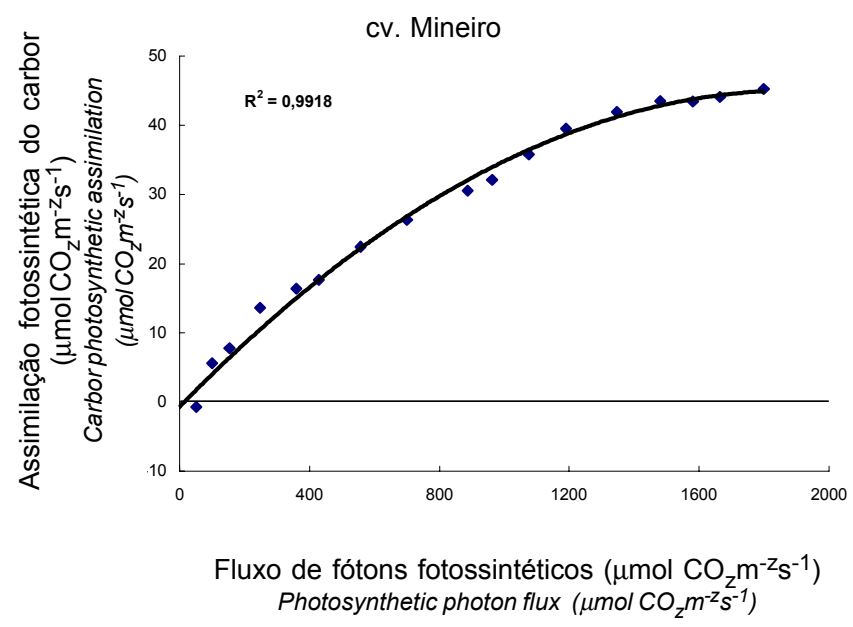

Figura 5 - Taxa de assimilação fotossintética do carbono em resposta ao fluxo de fótons, na cv. mineiro de capim-elefante cultivado em vaso, utilizando-se a luz solar como fonte luminosa.

Figure 5 - Carbon photosynthetic assimilation rate in response to photosynthetic photon flux in elephantgrass cv. mineiro, using sunlight as the light source.

Rev. bras. zootec., 30(6S):1975-1983, 2001 respiração e alta capacidade de acumulação de biomassa (WILSON e JONES, 1982). Nesse trabalho, os genótipos mineiro e CNPGL 91-27-5 tenderam aos mais baixos valores de taxa respiratória no escuro, respectivamente, 1,64 e $1,76 \mu \mathrm{mol} \mathrm{CO}_{2} \mathrm{~m}^{-2} \mathrm{~s}^{-1}$, enquanto o valor mais alto, foi observado no genótipo CNPGL 91-02-4, com 3,48 $\mu \mathrm{molCO}_{2} \mathrm{~m}^{-2} \mathrm{~s}^{-1}$ (Tabela 4). Embora os resultados de Rd não tenham apresentado correlação com a Amax, observou-se correlação positiva entre a $\mathrm{Rd}$ e a área foliar dos genótipos de capim-elefante (Figura 6).

Sob condições de luz saturante, pode-se esperar o aumento da relação ATP/ADP, o que poderia levar a uma disponibilidade máxima de ATP, sem, no entanto, provocar inibição da fosforilação oxidativa que ocorre nas cristas mitocondriais. Quanto maior o Rd, maior a quantidade de ATP para síntese de sacarose, consequentemente, para os processos metabólicos envolvendo o crescimento. PASSOS (1994), relatou que em plantas individuais a taxa respiratória é mais elevada em plantas $\mathrm{C} 4$ do que em $\mathrm{C}_{3}$, em amplitudes de temperatura de 12 a $42^{\circ} \mathrm{C}$. O autor ressaltou que esta tendência não é observada em dosséis e, talvez, a respiração não seja fator preponderante para a melhoria.

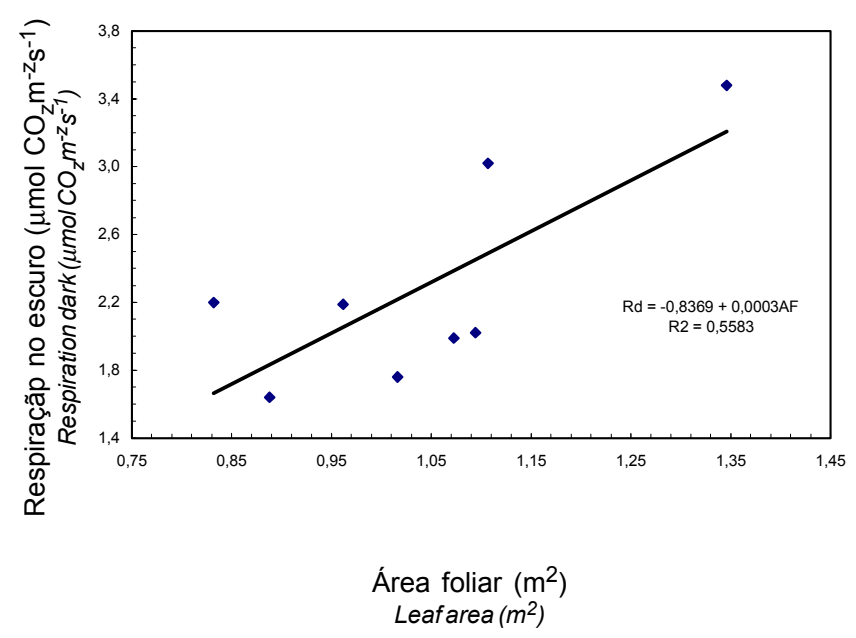

Figura 6 - Relação entre a área foliar e a taxa de respiração no escuro das folhas de oito genótipos de capimelefante.

Figure 6 - Relation between leaf area and dark respiration rate in imature leaves of eight genotypes of elephantgrass. 
Tabela 4 - Taxa respiratória $(R d)$, assimilação fotossintética de carbono $\left(A_{\max }\right)$ e relação $A_{\max } / R d$ em função da área foliar em oito genótipos de capim-elefante

Table 4 - Respiration dark rate $(R d)$, maximum carbon photosynthetic assimilation $\left(A_{\max }\right)$ and $A_{\max } / R d$ ratio compared to leaf area in eight genotypes of elephantgrass

\begin{tabular}{|c|c|c|c|c|}
\hline \multirow{2}{*}{$\begin{array}{l}\text { Genótipos } \\
\text { Genotypes }\end{array}$} & \multirow{2}{*}{$\begin{array}{l}\text { Área foliar }\left(\mathrm{m}^{2}\right) \\
\text { Leaf area }\left(\mathrm{m}^{2}\right)\end{array}$} & $\mathrm{Rd}$ & $\mathrm{A}_{\max }$ & \multirow{2}{*}{$\begin{array}{c}\text { Relação (Relation) } \\
\qquad \mathrm{A}_{\max } / \mathrm{Rd}\end{array}$} \\
\hline & & \multicolumn{2}{|c|}{$\left(\mu \mathrm{mol} \mathrm{CO} \mathrm{C}^{-2} \mathrm{~s}^{-1}\right)$} & \\
\hline Mineiro & 0,89 & 1,64 & 47,37 & 28,88 \\
\hline CNPGL91-01-2 & 1,09 & 2,02 & 39,05 & 19,33 \\
\hline CNPGL91-02-4 & 1,35 & 3,48 & 41,16 & 11,83 \\
\hline CNPGL91-19-1 & 1,07 & 1,99 & 39,64 & 19,92 \\
\hline Taiwan A-146 & 1,11 & 3,02 & 44,37 & 14,69 \\
\hline CNPGL 91-27-5 & 1,02 & 1,76 & 41,01 & 23,30 \\
\hline CNPGL91-27-1 & 0,96 & 2,19 & 40,81 & 18,64 \\
\hline CNPGL91-10-2 & 0,83 & 2,20 & 38,89 & 17,68 \\
\hline
\end{tabular}

\section{Conclusões}

O genótipo taiwan A-146 apresentou maiores teores de pigmentos fotossintéticos e curva de assimilação fotossintética mais pronunciada, revelando seu grande potencial fotossintético, sob condições de irradiância e temperatura não-limitantes.

A área foliar apresentou correlação positiva com a respiração no escuro obtida pelas curvas fotossintéticas, indicando a alta potencialidade dos genótipos, para a máxima produção de ATP, para os processos metabólicos envolvendo o crescimento.

\section{Referências Bibliográficas}

AUSTIN, R.B. 1989. Genetic variation in photosynthesis. J. Agric. Sci., 112:287-94.

AUSTIN, R.B., MORGAN, C.L., FORD, M.A. 1982. Flag leaf photosynthesis of Triticum aestivum and related diploid and tetraploid species. Ann. Bot., 149:64-68.

BARRO, F., GONZÁLES-FONTES, A., MALDONADO, J.M. 1996. Relation between photosynthesis and dark respiration in cereal leaves. J. Plant Physiology, 149:64-68.

BHAGSHARI, A.S., BROWN, R.H. 1986. Leaf photosynthesis and its correlation with leaf area. Crop Sci., 26:127-132.

BJÖRKMAN, O., DEMMING, B. 1987. Photon yield of $\mathrm{O}_{2}$ evolution and chlorophyll fluorescence characteristics at 77 $\mathrm{K}$ among vascular plants of diverse origins. Planta, 170:489-504.

CRISWELL, J.G., SHIBLES, R.M. 1971. Physiological basis for genotypic variation in net photosynthesis of oat leaves. Crop Sci., 11:550-553.

COOPER, J.P. 1970. Potencial production and energy conversion in temperate and tropical grasses. Herbage Abstracts, $40(1): 1-15$

DAHER, R.F. 1997. Diversidade morfológica e isozimática em capim-elefante (Pennisetum purpureum, Schum.). R. Bras. Zootec., 26(2):255-64.

DAHER, R.F., VASQUEZ, M.H., FERNANDES, A.M. et al. Competição de clones de capim-elefante (Pennisetum purpureum, Schum.) em Campos dos Goytacazes, RJ.
In: REUNIÃO ANUAL DE DA SOCIEDADE BRASILEIRA DE ZOOTECNIA, 34, 1997, Juiz de Fora. Anais... Juiz de Fora: SBZ, p.59-61. 1997.

EHLERINGER, J., BJÖRKMAN, O. 1977. Quantum yields for $\mathrm{CO}_{2}$ uptake in $\mathrm{C}_{3}$ and $\mathrm{C}_{4}$ plants, dependence on temperature, $\mathrm{CO}_{2}$ and $\mathrm{O}_{2}$ concentration. Plant Physiology, 59:86-90.

EUCLYDES, R.F. 1983. Sistema para análises estatísticas e genéticas (SAEG) - manual prático. Viçosa: CPD/UFV. $74 \mathrm{p}$.

FISCHER, R.A., REES, D., SAYRE, K.D. et al. 1998. Wheat yield associated with higher stomatal condutance and photosynthetic rate, and cooler canopies. Crop Sci., 38:1467-75.

GOMIDE, J.A. 1973. Fisiologia e manejo de plantas forrageiras. R. Soc. Bras. Zootec., 2(1):17-26.

GUTIERREZ, M., EDWARDS, G.E., BROWN, W.V. 1976. PEPcarboxilase containing species in the Brachiaria group of the family Panicoidae. Biochem. Syst. \& Ecol., 4:47-49.

HALL, D.O., RAO, K.K. 1994. Photosyntesis. Cambridge: University Press. 93p.

HEICHEL, G.H., MUSGRAVE, R.B. 1969. Varietal differences in net photosynthesis of Zea mays L. Crop Sci., 9:483-486.

HENDRY, G.A.F., PRICE, A.H. 1993. Stress indicators: chlorophylls and carotenoids. In: HENDRY, G.A.F., GRIME, J.P. (Eds.) Methods in comparative plant ecology. London: Chapman \& Hall. 485p.

KONNINGS, H. 1990. Physiological and morphological differences between plants with a high NAR or a high LAR as related to environmental conditions. In: LAMBERS et al. (Eds.) Causes and consequences of variation in growth rate and productivity. SBP: Academic Publishing. p.101-123.

LARCHER, W. 1995. Carbon utilization and dry matter production. Physiological plant ecology, 3.ed. Berlin: Springer. 506p

LUDLOW, M.M. 1985. Photosynthesis and dry matter production in $\mathrm{C} 3$ and $\mathrm{C} 4$ pasture plants, with emphasis on tropical C3 legumes and C4 grasses. Aust. J. Plant Physiology, 12:557-72.

MONTEITH, J.L. 1978. Reassessment of maximum growth rates for $\mathrm{C}_{3}$ and $\mathrm{C}_{4}$ crops. Exp. Agric., 14:1-5.

ORT, D.R., BAKER, N.R. 1988. Consideration of photosynthetic efficiency at low light as a major determinant of crop photosynthetic performance. Plant Physiology and Biochemistry, 26:555-65.

PASSOS, L.P. Estado do conhecimento sobre a fisiologia do capimelefante. In: SIMPÓSIO SOBRE CAPIM-ELEFANTE, 2,

Rev. bras. zootec., 30(6S):1975-1983, 2001 
1994, Juiz de Fora. Anais... Coronel Pacheco: EMBRAPACNPGL, p.12-56. 1994.

PAZ, L.G., MATOS, M.M.V.L. 1985. A fotossíntese e a produtividade das forrageiras. In: UNIVERSIDADE FEDERAL RURAL DE PERNAMBUCO, 1985, Recife. Anais... Recife, 1985, v.8, n.10, p.103-20.

SALISBURY, F.B., ROSS, C. W. 1991. Plant physiology. 3. ed. California, Belmont: Wadsworth Publishing Company. 692p.

TAIZ, L., ZEIGER, E. 1991. Plant physiology. 3.ed. California: The Benjamim-Cummings Publishing Company. 559p.

USUDA, H., KU, M.S.B., EDWARDS, G.E. 1984. Rates of photosynthesis relative to activity of photosynthetic enzimes, chlorophyll and soluble protein content among ten $\mathrm{C}_{4}$ species. Aust. J. Plant Physiology, 11:509-17.
WENG, J.H. 1984. Photosynthetic characteristics of Pennisetum purpureum Schum. and Digtaria decumbens Stent. Taiwan Livest. Res., 17:195-205.

WILSON, D., JONES, J.G. 1982. Effect of selection for dark respiration rate of mature leaves on crop yields of Lolium perenne cv. 523. Ann. Bot., 49:313-20.

ZELITCH, I. 1982. The close relationship between net photosyntesis and crop yield. Bioscience, 32:10.

Recebido em: 13/02/01

Aceito em: 31/07/01 